ORIGINAL ARTICLE

\title{
Prognostic significance of tenascin-C expression in superficial and invasive bladder cancer
}

\author{
A Brunner, C Mayerl, A Tzankov, I Verdorfer, I Tschörner, H Rogatsch, G Mikuz
}

J Clin Pathol 2004;57:927-931. doi: 10.1136/jcp.2004.016576

See end of article for authors' affiliations

....................

Correspondence to: A Brunner, Institute of Pathology, Innsbruck Medical Úniversity, Muellerstrasse 44, 6020 Innsbruck, Austria; andrea.brunner@ vibk.ac.at

Accepted for publication 23 March 2004

\begin{abstract}
Aims: Tenascin-C (Tn-C) is an extracellular matrix glycoprotein that is upregulated in malignant tumours. $\mathrm{Tn}-\mathrm{C}$ promotes cell growth, cell migration, and angiogenesis. It has been suggested to be a prognostic factor in various types of malignant tumours, but there is little information on its significance in bladder cancer with regard to overall survival (OS) and recurrence free survival (RFS).

Methods: Tn-C expression was studied in 106 patients with bladder cancer diagnosed between 1994 and 1997. Immunohistochemistry was performed using a monoclonal antibody against Tn-C. RFS and OS were estimated by the Kaplan-Meier method and compared by the log rank test in univariate analysis and by the Cox multistep regression method in multivariate analysis.

Results: Within the mean follow up period of 126 months, patients with diffuse $\mathrm{Tn}-\mathrm{C}$ staining in the tumour stroma had a significantly worse OS than those with negative staining or only moderate $\mathrm{Tn}$-C expression $(p=0.025)$. Patients with cytoplasmic expression of $\mathrm{Tn}-\mathrm{C}$ had a significantly better $\mathrm{OS}$ than those without $(p=0.001)$. Multivariate analysis, taking into consideration age, grade, stage, tumour associated carcinoma in situ, progression, and $\mathrm{Tn}-\mathrm{C}$ staining in tumour stroma, showed that only expression of $\mathrm{Tn}-\mathrm{C}$ in invasive tumour cells was an independent positive prognostic factor for OS ( $p=0.049$ ).

Conclusions: Tn-C may provide important prognostic information in bladder cancer depending on the expression pattern in the tumour stroma or cytoplasm of the tumour cells.
\end{abstract}

$\mathrm{T}$ enascin-C (Tn-C) is an extracellular matrix glycoprotein that is expressed transiently during embryogenesis. ${ }^{12}$

The expression of Tn-C is downregulated in most adult tissues, but reappears in various pathological conditions, including reparative, hyperplastic, inflammatory, and neoplastic processes. ${ }^{3}{ }^{4}$ Functionally, Tn-C interacts with fibronectin and can be defined as an anti-adhesive or adhesion modulating protein. ${ }^{35}$ Several studies have linked Tn-C expression in malignant tumours to increased invasive and metastastic potential. ${ }^{3-10}$

Bladder cancer is the most common malignant tumour of the urogenital tract, with most being transitional cell carcinomas (TCCs)..$^{11}$ Most patients with TCC initially present with superficial tumours (pTa and pTl), but the disease progresses in approximately $25 \% .{ }^{13}$ About $20 \%$ of patients have muscle invasive disease at first presentation. ${ }^{12}$ A wide range of markers has been tested to help identify those patients who will develop progression or are at high risk for metastasis. ${ }^{13} 14$

"Functionally, tenascin-C interacts with fibronectin and can be defined as an anti-adhesive or adhesion modulating protein"

Tn-C has been suggested to be of prognostic relevance in malignant tumours. ${ }^{8}{ }^{15-19}$ Cytoplasmic Tn-C expression was found in superficial cells of normal urothelium, but rarely in bladder cancer. ${ }^{20}{ }^{21}$ Expression of Tn-C has been described in the stroma of invasive TCC of the urinary bladder and in the fibrovascular cores, suggesting an association with muscle invasion and neoangiogenesis. ${ }^{22} 23$ To assess further the expression and prognostic relevance of Tn-C in superficial and invasive bladder cancer, we performed a retrospective study of 106 patients with a mean clinical follow up of 126 months.

\section{MATERIAL AND METHODS}

One hundred and six cases of TCC of the bladder diagnosed between January 1994 and December 1997 were selected from the files of the institute of pathology at the Innsbruck Medical University, Austria; the clinical data were available for all these cases and they were all primary diagnoses, with no previous surgical or neoadjuvant cytoreductive treatment. All specimens were obtained by means of transurethral resection. The depth of bladder wall infiltration of all seven pT3 and pT4 tumours was corroborated by the stage obtained on the consecutive cystectomy specimens. Disease recurrence was defined as the detection of a newly developed tumour more than one month after primary resection. Disease progression was defined as an increase of stage and/or grade in a recurrent tumour.

For immunohistochemistry, a monoclonal antibody against Tn-C (clone T2H5; Neomarkers, Freemont, California, USA) was used at a dilution of $1 / 50$. After dewaxing with xylol and rehydration with graded ethanol, antigen retrieval was carried out by treating sections with $0.1 \mathrm{mM}$ EDTA buffer $(\mathrm{pH} 8.0)$ in a microwave oven $(750 \mathrm{~W})$ for 10 minutes. Afterwards, the slides were incubated with $1.5 \% \mathrm{H}_{2} \mathrm{O}_{2}$ in methanol. To block non-specific binding, the sections were also treated with a blocking solution (blocking reagent; Boehringer, Mannheim, Germany) for 45 minutes. Sections were then incubated with the primary antibody overnight at $4^{\circ} \mathrm{C}$. After washing, the reaction was visualised using the alkaline phosphatase anti-alkaline phosphatase method (Dako, Glostrup, Denmark) and Fast red (Serotec, Kiddington, Oxford, UK) as substrate. For positive control experiments, a squamous cell carcinoma of the larynx was

Abbreviations: CIS, carcinoma in situ; OS, overall survival; RFS, recurrence free survival; TCC, transitional cell carcinoma; $\mathrm{Tn}-\mathrm{C}$, tenascin-C 
used and for negative control experiments non-relevant IgG was applied instead of the specific antibody.

Tn-C staining was assessed by scoring the tumours semiquantitatively as,+++ , and +++ , corresponding to focal, multifocal, and diffuse immunoreactivity, respectively. Stromal staining in invasive tumours, and cytoplasmic staining in superficial, intermediate/basal, and invasive tumour cells was evaluated. Adjacent normal urothelium and the underlying lamina propria, in addition to sections from normal urothelium, were used as controls.

SPSS 11.0 for Windows was used for statistical analysis. Overall survival (OS) and recurrence free survival (RFS) were analysed by the Kaplan-Meier method and compared by the $\log$ rank test. The Pearson $\chi^{2}$ test was used to test correlations between Tn-C expression and recurrence rate, grade, and stage of disease. Multivariate analysis was performed to identify independent prognostic markers for OS using a Cox multistep regression model. A p value $<0.05$ was considered significant.

\section{RESULTS}

Our study group consisted of $74(70 \%)$ male and $32(30 \%)$ female patients. The mean age was 73 years (range, 43-100). Histopathologically, the group consisted of 25 pTa (11 grade 1,12 grade 2 , and two grade 3 tumours), 39 pTl (one grade 1 , 15 grade 2 , and 23 grade 3 tumours), 36 pT2-4 (eight grade 2 and 28 grade 3 tumours), five carcinomas in situ (CIS) and one papilloma.

Fifty eight $(55 \%)$ patients had recurrent disease, with a mean number of two recurrences (range, one to nine). Fifteen (14\%) patients had a tumour associated CIS at initial presentation. In $21(20 \%)$ patients disease progressed. Within the follow up period, there were 34 malignancy related deaths. Ten patients died of a secondary neoplasm, eight of cardiovascular disease, and three of other causes, including pulmonary disease and trauma.

Stromal Tn-C staining and staining in invasive tumour cells were evaluated in all 75 invasive tumours included in the study; weak Tn-C expression in the lamina propria of non-invasive tumours was regarded as normal, ${ }^{21}$ and was used as an internal positive control. Stromal Tn-C expression was detected in 56 of the 75 invasive tumours and in the tumour cells of the invasive front of 35 . Tn-C expression in superficial and intermediate cells was evaluated in 84 tumours (25 pTa, 36 pTl, 17 pT2 5 CIS, and one papilloma), irrespective of the grade or stage of disease, when the superficial portion was present. Staining of the superficial cells was seen in 59 of the 75 tumours, and staining of the intermediate/basal cells was seen in 38 (fig 1). Staining in the fibrovascular cores was found in 28 of all 106 specimens; namely, eight pTa, 14 pT1, and six pT2 tumours.

Tn-C staining in the tumour stroma correlated positively with disease stage, whereas staining in invasive tumour cells correlated negatively $(\mathrm{p}=0.006$ and $\mathrm{p}=0.007$, respectively). There was also a negative correlation between Tn-C expression in the stroma and in the invasive tumour cells $(\mathrm{p}=0.007)$. No such correlation was seen for the expression of Tn-C in the superficial or intermediate/basal cells of papillary bladder tumours. There was also no correlation between Tn-C expression and tumour grade.

Patients with diffuse Tn-C staining of the tumour stroma had a significantly worse OS ( 14 of the 21 patients died) than those with only focal and multifocal staining ( 11 of 22 died) and those without staining (nine of 32 died; $p=0.025$; fig 2).

When compared by the log rank test, patients with cytoplasmic Tn-C expression had a significantly better OS ( 18 of 72 patients died) than Tn-C negative patients ( 19 of 34 died; $p=0.0012$ ). In addition, patients with Tn-C expression in the superficial cells had a considerably better OS ( 11 of 59 patients died) than those without TN-C expression in the superficial cells ( 12 of 25 died; $p=0.0037$; fig 3 ). A similar result was seen for patients with cytoplasmic staining of invasive cells. Patients with diffuse or multifocal cytoplasmic staining in invasive tumour cells had a better OS than those without or with only focal staining, although it was only of borderline significance $(p=0.5)$. Apart from Tn-C staining the only factors that significantly influenced OS were stage $(p=0.0003)$ and grade $(p=0.0039)$.

No influence of Tn-C expression on the number of recurrences or RFS was detected in either the stroma or in the cytoplasm of the tumour cells. The only factor that
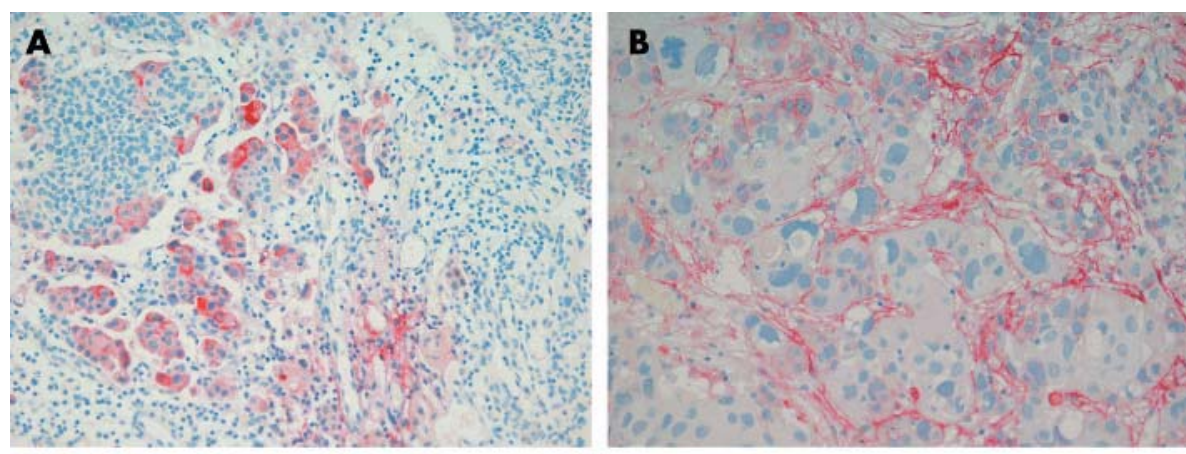

Figure 1 (A) Low power view of invasive bladder cancer with extensive cytoplasmic tenascin-C (Tn-C) expression and scarce stromal staining (original magnification, $\times 200$ ). High power view of (B) stromal and (C) cytoplasmic $\mathrm{Tn}-\mathrm{C}$ positivity in invasive cancer and (D) $\mathrm{Tn}-\mathrm{C}$ expression in the superficial cells of a papillary bladder cancer (original magnification, $\times 400$ ).
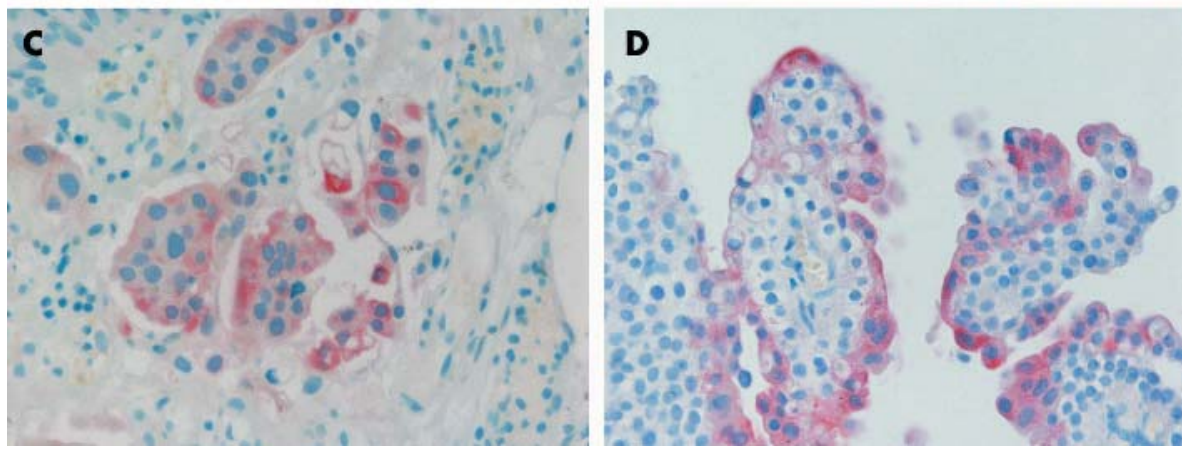
significantly correlated with RFS was disease progression: there were higher numbers of recurrences and shorter intervals to recurrence in patients with disease progression (20 relapses in 21 patients; RFS, 35 months) compared with those without progression (35 of 76; RFS, 141 months; $\mathrm{p}<0.0001$ for both).

Multivariate analysis taking into account age, stage, grade, tumour associated CIS, progression, and Tn-C staining in stroma and invasive cells revealed that Tn-C staining in invasive tumour cells was an independent positive prognostic factor for OS (table 1).

\section{DISCUSSION}

Our analysis of Tn-C expression in 106 urothelial carcinomas with a mean follow up period of 126 months showed that the pattern and localisation of staining of the tumour cells and stroma has prognostic significance. Invasive tumours with diffuse Tn-C staining in the stroma had a significantly worse prognosis than those that were negative or stained weakly. Because few data on the prognostic significance of Tn-C expression in bladder cancer are available, our data can only be compared with the results found in studies on other cancer types. ${ }^{20} 23$ There are striking differences among different cancer types; in some, Tn-C stromal positivity was a sign of poor prognosis, ${ }^{278}{ }^{15-18}$ whereas in others it was predictive of a better outcome. ${ }^{192-26}$ Even in the same cancer types (for example, breast and colon), positive Tn-C stromal staining was associated with a poor outcome in some studies $^{15} 816$ and a good prognosis in others. ${ }^{24} 25$ Moreover, some authors found no correlations between Tn-C stromal expression and outcome. ${ }^{27} 28$ One possible reason for these

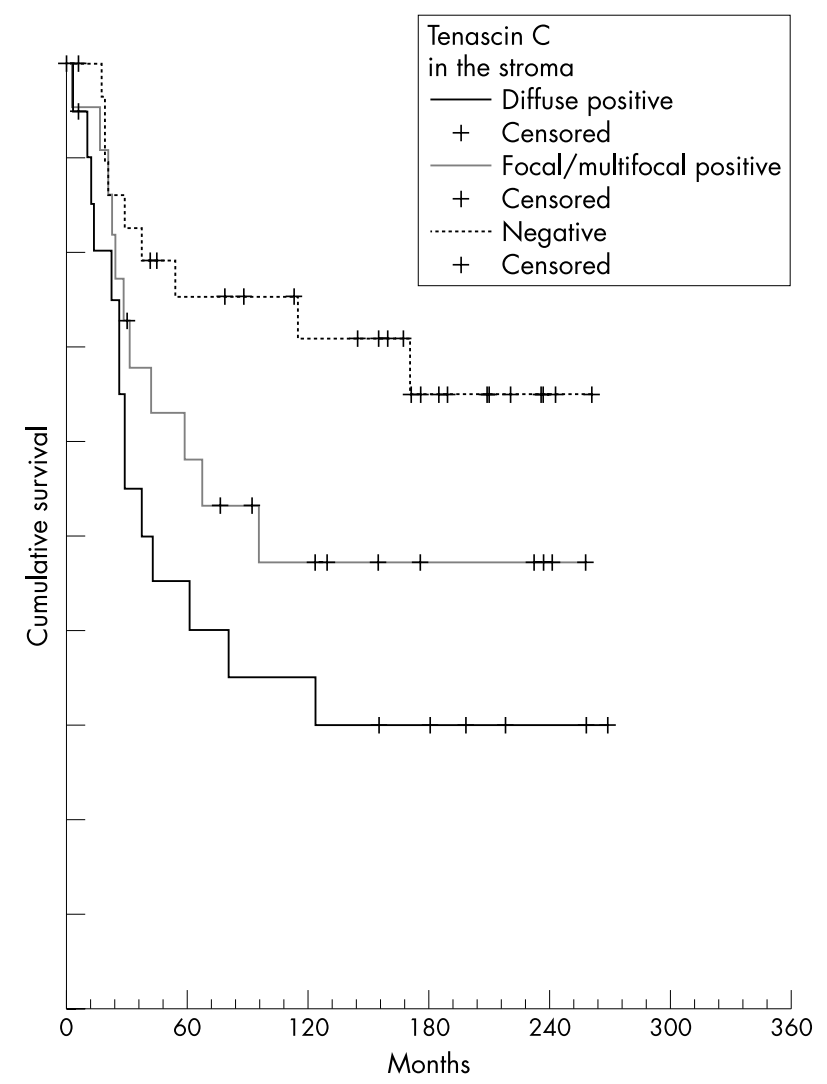

Figure 2 Overall survival of patients correlated with tenascin- $C(T n-C)$ staining in tumour stroma. $\mathrm{Tn}-\mathrm{C}$ diffuse positive versus $\mathrm{Tn}-\mathrm{C}$ focal/ multifocal positive and versus $\mathrm{Tn}-\mathrm{C}$ negative cases compared by the log rank test.

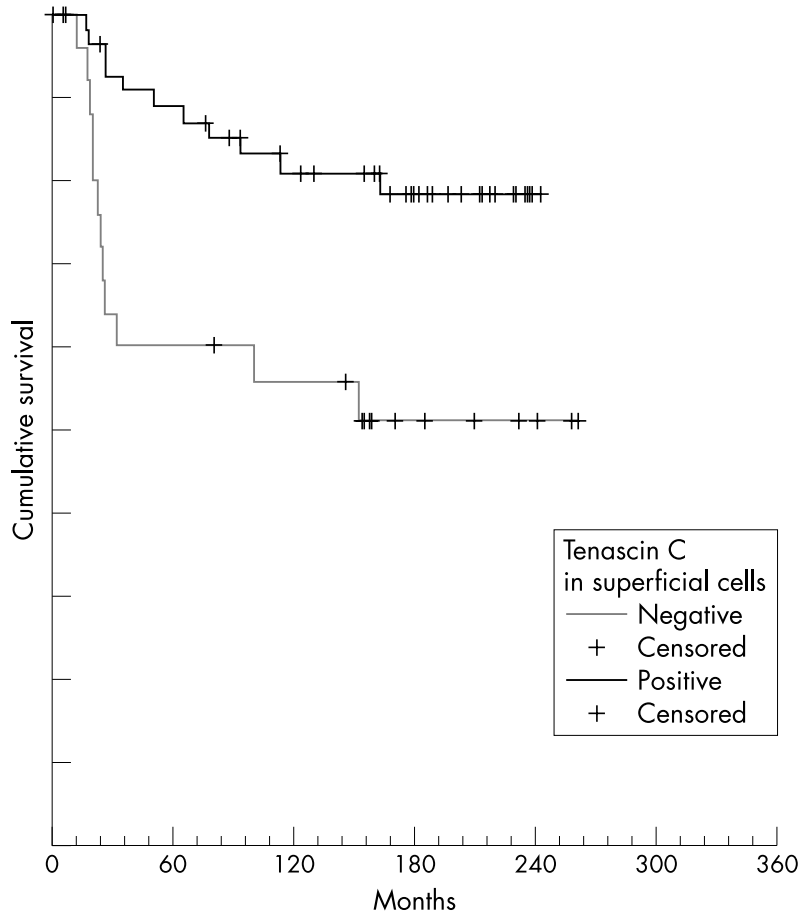

Figure 3 Overall survival of patients correlated with the expression of tenascin- $C(T n-C)$ in superficial cells. $T n-C$ positive versus $T n-C$ negative cases compared by the log rank test.

contradictory results could be the use of different parameters for the assessment of the biological behaviour of the tumours. In some cases, the prognostic importance of Tn-C has been analysed by comparing staining with survival rates. ${ }^{815} 161819$ However, in other reports, Tn-C expression has been compared only with morphological predictors of worse prognosis, such as vascular invasion and lymph node metastases. ${ }^{27} 152627$

\section{"Tenascin-C expression in bladder cancer may provide additional prognostic information with regard to patient outcome"}

In addition to stromal Tn-C expression, we saw cytoplasmic staining in superficial and invasive tumour cells. It is well known that not only stromal fibroblasts but also tumour cells are a source of Tn-C. ${ }^{216-18} 2930$ In our group, patients with staining in superficial cells had a better OS than those with loss of Tn-C expression. Normal urothelial cells are able to produce large amounts of Tn-C in vitro and superficial cells of

Table 1 Multivariate analysis for the independent prognostic value of expression of $\mathrm{Tn}-\mathrm{C}$ in invasive bladder cancer

\begin{tabular}{lll}
\hline Factor & Relative risk & p Value \\
\hline Grade & 8.8 & $0.003^{*}$ \\
Progression & 5.2 & $0.02^{*}$ \\
Tn-C invasive cells & 4.6 & $0.03^{*}$ \\
Stage & 3.2 & 0.07 \\
Tumour associated CIS & 2 & 0.16 \\
Tn-C stroma & 1.2 & 0.26 \\
Age & 1.1 & 0.29 \\
\hline
\end{tabular}

*Significant.

CIS, carcinoma in situ; $\mathrm{Tn}-\mathrm{C}$, tenascin-C. 


\section{Take home messages}

- Patients with diffuse tenascin-C (Tn-C) staining in the tumour stroma had a significantly worse overall survival (OS) than those with negative or moderate $\mathrm{Tn}-\mathrm{C}$ expression

- Patients with cytoplasmic expression of $\mathrm{Tn}-\mathrm{C}$ had a significantly better OS than those without

- Only expression of $\mathrm{Tn}-\mathrm{C}$ in invasive tumour cells was an independent positive prognostic factor for OS in multivariate analysis

- Tn-C may provide important prognostic information in bladder cancer depending on the expression pattern in the tumour stroma or cytoplasm of the tumour cells

normal urothelium have been shown to express Tn-C. ${ }^{20}$ In the bladder, the expression of Tn-C seems to be a normal function of superficial cells, and loss of superficial staining might be a sign of dedifferentiation. ${ }^{20}$ Tn-C expression in invasive tumour cells was seen in several tumour types, with similar conflicting results concerning tumour behaviour. $^{2}{ }^{17} 1829$ Some authors detected a less favourable prognosis in patients with cytoplasmic staining, suggesting that $\mathrm{Tn}-\mathrm{C}$ is promptly released into the stroma, and may stimulate cells in some sort of autocrine fashion. ${ }^{29}$ These results are supported by the fact that the amount of Tn-C synthesis detected by in situ hybridisation for mRNA exceeds that detected by immunohistochemistry. ${ }^{2}$ Others reported that cytoplasmic staining in invasive tumour cells inversely correlates with lymph node metastasis, suggesting that Tn-C expression by neoplastic cells is an early event representing a less aggressive phenotype. ${ }^{16}{ }^{24}$ We found that cytoplasmic staining was associated with significantly lower stromal expression and that Tn-C in invasive tumour cells was an independent positive prognostic factor, supporting the assumption that tumours with cytoplasmic staining might behave less aggressively. Several studies suggest that the migration and adhesion of tumour cells strongly depend on both the composition and concentration of extracellular matrix molecules. ${ }^{51-33}$ Yokosaki and co-authors showed that different integrin receptors expressed in a colon cancer cell line can produce greatly differing effects on the proliferative response to Tn-C. ${ }^{34}$ In addition, the structure and size of Tn-C varies as a result of alternative splicing of the exons within the FNIII repeat domain, generating functional diversity with different effects on cell proliferation and migration. ${ }^{356}$ Adams et al found that different isoforms of Tn-C are expressed in breast cancer and that two isoforms (Tn16 and Tn14/16) may be helpful in predicting invasion. ${ }^{36}$ Interestingly, in situ hybridisation showed that both isoforms were exclusively synthesised in the stromal compartment. These findings are in contrast to previous reports relating cytoplasmic Tn-C expression to a more aggressive phenotype, 2936 but confirm the possibility that stromal and cytoplasmic Tn-C expression may have different effects on tumour behaviour, as suggested by our study. ${ }^{16}{ }^{24}$

In conclusion, Tn-C expression in bladder cancer may provide additional prognostic information with regard to patient outcome, although its role in the tumorigenesis and progression of bladder cancer still needs to be investigated.

\section{ACKNOWLEDGEMENTS}

We thank H Muehlboeck from the Cancer Registry of Tyrol/Austria, Institute for Epidemiology for providing epidemiological data.

\section{Authors' affiliations}

A Brunner, A Tzankov, I Verdorfer, I Tschörner, H Rogatsch, G Mikuz, Institute of Pathology, Innsbruck Medical University, Muellerstrasse 44, 6020 Innsbruck, Austria

Mayerl C, Institute of Pathophysiology, Innsbruck Medical University

\section{REFERENCES}

1 Chiquet-Ehrismann R, Mackie EJ, Pearson CA, et al. Tenascin: an extracellular matrix protein involved in tissue interactions during fetal development and oncogenesis. Cell 1986;47:131-9.

2 Ishihara A, Yoshida T, Tamaki $\mathrm{H}$, et al. Tenascin expression in cancer cells and stroma of human breast cancer and its prognostic significance. Clin Cancer Res 1995; 1:1035-341.

3 Chiquet-Ehrismann R, Chiquet $M$. Tenascin: regulation and putative functions during pathological stress. J Pathol 2003;200:488-99.

4 Koukoulis GK, Gould VE, Bhattacharyya A, et al. Tenascin in normal, reactive, hyperplastic and neoplastic tissues: biological and pathological implications. Hum Pathol 1991 ;22:636-43.

5 Chiquet-Ehrismann R, Kalla P, Pearson CA, et al. Tenascin interferes with fibronectin action. Cell 1988;53:383-90.

6 Jahkola T, Toivonen T, Nordling S, et al. Expression of tenascin-C in intraductal carcinoma of human breast: relationship to invasion. Eur J Cancer 1998;34: 1687-92.

7 Jahkola T, Toivonen T, Virtanen I, et al. Tenascin- $C$ expression in invasion border of early breast cancer: a predictor of local and distant recurrence. Br J Cancer 1998;78:1507-13.

8 Emoto K, Yamada Y, Sawada H, et al. Annexin overexpression correlates with stromal tenascin-C overexpression. A prognostic marker in colorectal cancer. Cancer 2001;92:1419-26

9 Salmenkivi K, Haglund C, Arola J, et al. Increased expression of tenascin in pheochromocytomas correlates with malignancy. Am J Surg Pathol 2001;25:1429-3.

10 Regezi JA, Ramos DM, Pytela R, et al. Tenascin and $\beta 6$ integrin are overexpressed in floor of mouth in situ carcinomas and invasive squamous cell carcinomas. Oral Oncol 2002;38:332-6.

11 Brauers A, Jakse G. Epidemiology and biology of human urinary cancer. $J$ Cancer Res Clin Oncol 2000;126:575-83.

12 Al-Sukhun S, Hussain M. Current understanding of the biology of advanced bladder cancer. Cancer 2003;97(suppl 8):2064-75.

13 Kausch I, Böhle A. Molecular aspects of bladder cancer III. Prognostic markers of bladder cancer. Eur Urol 2002;41:15-29.

14 Borden LS Jr, Clark PE, Hall MC. Bladder cancer. Curr Opin Oncol 2003; 15:227-33.

15 Kressner U, Lindmark G, Tomasini-Johansson B, et al. Stromal tenascin distribution as a prognostic marker in colorectal cancer. $\mathrm{Br} J$ Cancer 1997;76:526-30.

16 loachim E, Charchanti A, Briasoulis E, et al. Immunohistochemical expression of extracellular matrix components tenascin, fibronectin, collagen type IV and laminin in breast cancer: their prognostic value and role in tumour invasion and progression. Eur J Cancer 2002;38:2362-70.

17 Aishima S, Taguchi K, Terashi T, et al. Tenascin expression at the invasive front is associated with poor prognosis in intrahepatic cholangiocarcinoma. Mod Pathol 2003;16:1019-27.

18 Kaarteenaho-Wiik R, Soini Y, Pöllänen R, et al. Over-expression of tenascin- $\mathrm{C}$ in malignant pleural mesothelioma. Histopathology 2003;42:280-91.

19 Wiksten JP, Lundin J, Nordling S, et al. Tenascin-C expression correlates with prognosis in gastric cancer. Oncology 2003;64:245-50.

20 Booth C, Harnden P, Selby PJ, et al. Towards defining roles and relationships for tenascin- $C$ and TGF $\beta-1$ in the normal and neoplastic urinary bladder. J Pathol 2002; 198:359-68.

21 Wilson CB, Leopard J, Cheresh DA, et al. Extracellular matrix and integrin composition of the normal bladder wall. World J Urol 1996; 14:30-7.

22 Deen S, Ball RY. Basement membrane and extracellular interstitial matrix components in bladder neoplasia-evidence of angiogenesis. Histopathology 1994;25:475-81.

23 Tiitta O, Wahlstrom T, Virtanen I, et al. Tenascin in inflammatory conditions and neoplasms of the urinary bladder. Virchows Arch B Cell Pathol Incl Mol Pathol 1993;63:283-7.

24 Sugawara I, Hirakoshi J, Masunaga A, et al. Reduced tenascin expression in colonic carcinoma with lymphogenous metastasis. Invasion Metastasis $1991 ; 11: 325-31$

25 Shoji T, Kamiya T, Tsubara A, et al. Tenascin staining positivity and the survival of patients with invasive breast carcinoma. J Surg Res 1993;55:295-7.

26 Buyukbayram H, Arslan A. Value of tenascin-C content and association with clinicopathological parameters in uterine cervical lesions. Int J Cancer 2002; 100:719-22.

27 Moch $\mathrm{H}$, Torhorst J, Durmuller U, et al. Comparative analysis of the expression of tenascin and established prognostic factors in human breast cancer. Pathol Res Pract 1993; 198:510-14.

28 Atula $T$, Hedstrom J, Finne $P$, et al. Tenascin- $C$ expression and its prognostic significance in oral and pharyngeal squamous cell carcinoma. Anticancer Res 2003;23(3C):3051-6 
29 Yoshida $\mathrm{T}$, Yoshimura $\mathrm{E}$, Numata $\mathrm{H}$, et al. Involvement of tenascin- $\mathrm{C}$ in proliferation and migration of laryngeal carcinoma cells. Virchows Arch 1999;435:496-500.

30 Tôkés AM, Paku S, Tóth S, et al. Tenascin expression in primary and recurrent breast carcinomas and the effect of tenascin on breast tumour cell cultures. Pathol Oncol Res 2000;6:202-9.

31 Huang W, Chiquet-Ehrismann R, Moyano JV, et al. Interference of tenascin with syndecan- 4 binding to fibronectin blocks cell adhesion and stimulates tumor cell proliferation. Cancer Res 2001;61:8586-94.

32 Hauptmann S, Budianto D, Denkert $C$, et al. Adhesion and migration of HRT18 colorectal carcinoma cells on extracellular matrix components typical for the desmoplastic stroma of colorectal adenocarcinomas. Oncology 2003;65:174-81.
33 Kalembeyi I, Inada $\mathrm{H}$, Nishiura $\mathrm{R}$, et al. Tenascin- $\mathrm{C}$ upregulates matrix metalloproteinase- 9 in breast cancer cells: direct and synergistic effects with transforming growth factor $\beta 1$. Int J Cancer 2003;105:53-60

34 Yokosaki $Y$, Monis $H$, Chen J, et al. Differential effects of the integrins $\alpha 9 \beta 1$, $\alpha \vee \beta 3$ and $\alpha \vee \beta 6$ on cell proliferative responses to tenascin. J Biol Chem 1996;271:24144-50.

35 Tsunoda T, Inada H, Kalembeyi I, et al. Involvement of large tenascin-C splice variants in breast cancer progression. Am J Pathol 2003; 162:1857-67.

36 Adams M, Jones JL, Walker RA, et al. Changes in tenascin-C isoform expression in invasive and preinvasive breast disease. Cancer Res $2001 ; 62: 3289-97$.

\section{Clinical Evidence-Call for contributors}

Clinical Evidence is a regularly updated evidence based journal available worldwide both as a paper version and on the internet. Clinical Evidence needs to recruit a number of new contributors. Contributors are health care professionals or epidemiologists with experience in evidence based medicine and the ability to write in a concise and structured way.

\section{Currently, we are interested in finding contributors with an interest in} the following clinical areas:

Altitude sickness; Autism; Basal cell carcinoma; Breast feeding; Carbon monoxide poisoning; Cervical cancer; Cystic fibrosis; Ectopic pregnancy; Grief/bereavement; Halitosis; Hodgkins disease; Infectious mononucleosis (glandular fever); Kidney stones; Malignant melanoma (metastatic); Mesothelioma; Myeloma; Ovarian cyst; Pancreatitis (acute); Pancreatitis (chronic); Polymyalgia rheumatica; Post-partum haemorrhage; Pulmonary embolism; Recurrent miscarriage; Repetitive strain injury; Scoliosis; Seasonal affective disorder; Squint; Systemic lupus erythematosus; Testicular cancer; Varicocele; Viral meningitis; Vitiligo However, we are always looking for others, so do not let this list discourage you.

\section{Being a contributor involves:}

- Appraising the results of literature searches (performed by our Information Specialists) to identify high quality evidence for inclusion in the journal.

- Writing to a highly structured template (about 2000-3000 words), using evidence from selected studies, within 6-8 weeks of receiving the literature search results.

- Working with Clinical Evidence Editors to ensure that the text meets rigorous epidemiological and style standards.

- Updating the text every eight months to incorporate new evidence.

- Expanding the topic to include new questions once every 12-18 months.

If you would like to become a contributor for Clinical Evidence or require more information about what this involves please send your contact details and a copy of your CV, clearly stating the clinical area you are interested in, to Claire Folkes (cfolkes@bmigroup.com).

\section{Call for peer reviewers}

Clinical Evidence also needs to recruit a number of new peer reviewers specifically with an interest in the clinical areas stated above, and also others related to general practice. Peer reviewers are health care professionals or epidemiologists with experience in evidence based medicine. As a peer reviewer you would be asked for your views on the clinical relevance, validity, and accessibility of specific topics within the journal, and their usefulness to the intended audience (international generalists and health care professionals, possibly with limited statistical knowledge). Topics are usually 2000-3000 words in length and we would ask you to review between 2-5 topics per year. The peer review process takes place throughout the year, and our turnaround time for each review is ideally 10-14 days.

If you are interested in becoming a peer reviewer for Clinical Evidence, please complete the peer review questionnaire at www.clinicalevidence.com or contact Claire Folkes(cfolkes@bmigroup.com). 